\title{
Factors promoting engaged exploration with computer simulations
}

\author{
Noah S. Podolefsky, * Katherine K. Perkins, and Wendy K. Adams \\ Department of Physics, University of Colorado at Boulder, Boulder, Colorado 80309-0390, USA
}

(Received 10 February 2010; published 5 October 2010)

\begin{abstract}
This paper extends prior research on student use of computer simulations (sims) to engage with and explore science topics, in this case wave interference. We describe engaged exploration; a process that involves students actively interacting with educational materials, sense making, and exploring primarily via their own questioning. We analyze interviews with college students using PhET sims in order to demonstrate engaged exploration, and to identify factors that can promote this type of inquiry. With minimal explicit guidance, students explore the topic of wave interference in ways that bear similarity to how scientists explore phenomena. PhET sims are flexible tools which allow students to choose their own learning path, but also provide constraints such that students' choices are generally productive. This type of inquiry is supported by sim features such as concrete connections to the real world, representations that are not available in the real world, analogies to help students make meaning of and connect across multiple representations and phenomena, and a high level of interactivity with real-time, dynamic feedback from the sim. These features of PhET sims enable students to pose questions and answer them in ways that may not be supported by more traditional educational materials.
\end{abstract}

DOI: 10.1103/PhysRevSTPER.6.020117

PACS number(s): 01.40.- d, 01.50.H-, 01.50.Lc

\section{INTRODUCTION}

Over the last decade, new technologies have made rich and dynamic visual representations possible on common personal computers. Previously, the power of typical computers available in schools severely limited the range of computerbased educational experiences available to students. Educational simulations of this prior era were often based on simple line drawings, and the degree of interactivity was usually limited to setting one or two parameters and then watching a resulting animation. Modern computational power allows much more complex models, higher fidelity visual representations, and gives users of educational simulations the control and flexibility to make changes and see the effects in real time. With these advances, simulations can provide students with opportunities for rich and dynamic educational experiences as well as instantaneous feedback on the results of a virtual "experiment." In particular, the activities that students engage in with modern computer simulations can be quite different from those in traditional educational environments. Sims provide students with access to questions and methods of inquiry which are well aligned with the ways scientists use experiments for exploration and discovery. Prior work has shown that children can engage in productive inquiry and exploration along these lines with computer simulations [1-3]. In this paper, we target college students and characterize in detail the type of engagement that is possible when these students explore physical phenomena with computer simulations.

This paper builds on prior research with PhET computer simulations ("sims"). PhET sims are a substantial ( 85) and growing suite of computer simulations for engaging students with science content. The sims are freely distributed from the PhET website [4], with roughly 10 million uses in the past

\footnotetext{
*Corresponding author; noah.podolefsky@ colorado.edu
}

year. The majority of PhET sims are for teaching physics, but there are a growing number in chemistry, biology, and other sciences. Considerable research has investigated the use of $\mathrm{PhET}$ sims in a variety of educational settings [5-8]. Interactivity in computer simulations is known to have educational benefits $[9,10]$, but there is a broad range in the degree of interactivity across existing simulations. PhET sims offer a high degree of interactivity in terms of user control, dynamic feedback, and use of multiple representations. Sims also provide balanced challenges, implicit puzzles that are challenging but attainable, depending on the level of the student, and thereby promote student inquiry $[11,12]$. This paper builds on prior research to examine in more detail the way students engage with sims.

In particular the focus is along two themes-engaged exploration [13] and analogy [14-17]. Engaged exploration is a process that involves students actively interacting with educational materials, sense making, and exploring largely via their own questioning. PhET sims are designed to support and promote engaged exploration, and in this paper we demonstrate the effectiveness of sims in achieving this goal. Analogy, broadly stated, is a cognitive tool that uses familiar ideas to make sense of and generate new ideas about an unfamiliar topic [18-20]. We investigate students' use of analogy in the context of using a PhET sim. Our findings suggest that the use of analogy is a key component of student sense making in use of the sims, evident in the ways students draw on familiar ideas to help them generate ideas about the new and unfamiliar topics they encounter in the sim. With educational tools that are sufficiently engaging and provide appropriate scaffolding, students may generate analogies to make sense of physical phenomena.

In this study, we are primarily interested in understanding the process of student engagement and use of analogy. We analyze individual interviews of students using a sim, specifically (1) characterizing student engagement that is possible with PhET sims, (2) demonstrating student use of analogy with sims, and (3) focusing on interaction with sims as a 


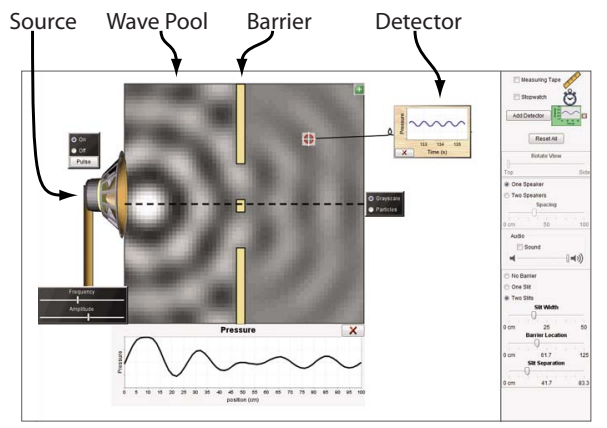

FIG. 1. (Color) Sound tab of Wave Interference. Each tab uses the same basic layout. The source (here a loud speaker) can alternatively be a faucet (water wave) or flashlight (light wave). The gray wave shown here can represent a water wave, sound wave, or light wave. The source, wave color, and graph labels change depending on the phenomenon represented. The graph at the bottom shows the wave along the dashed line in the pool. A barrier with one or two slits can be placed in the wave pool. A detector is used to measure the wave activity at different points in the pool.

process of building connections through exploration.

The next section describes the sim that was used in this study: Wave Interference. We then outline the theoretical framework for analyzing data from interviews with students using sims, followed by the methodology and results of the interviews which demonstrate student engaged exploration and use of analogy with the sim.

\section{WAVE INTERFERENCE SIM}

The Wave Interference sim is designed to allow students to explore interference in the context of water waves, sound waves, and light waves. It is currently the eighth most popular sim on the PhET website. Many of the sim's design features are based on general guidelines used for all PhET sims [5], including a high level of interactivity, an intuitive user interface, and use of representations that draw on students' existing ideas. In addition to these general guidelines, the design of Wave Interference makes explicit use of analogies, as described below

The sim is divided into three panels, or "tabs," one for each wave phenomenon (water, sound, and light). Each tab uses the same basic layout (Fig. 1). This layout uses concentric circles (ripples view) to represent the wave in an area called the wave pool. The circles are blue for water, gray for sound, and vary in color for light, depending on the wavelength of light selected. The frequency and amplitude of the wave can be adjusted with sliders to the left of the pool, and various other adjustments can be made in the "control panel" on the right. The water wave can be rotated to a side view, which shows a cross section of a water wave oscillating up and down. [Fig. 2(a)] The side view serves as a second representation of water meant to help students make the realworld connection that the circular shapes in the ripples view depict water waves moving up and down. In the sound tab, users may select grayscale view, as shown in Fig. 1, or particles view [Fig. 2(b)], which depicts air particles moving back and forth. In the light tab, users can activate a screen on
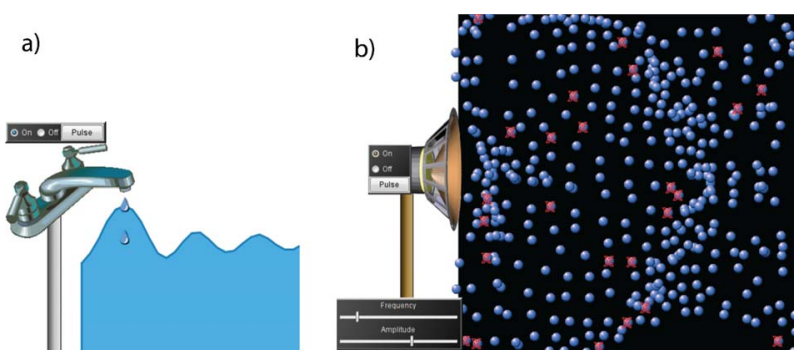

FIG. 2. (Color) (a) Side view of water wave. (b) Particles view of sound wave

the right side of the pool. This screen shows the light pattern that falls on it-if light is interfering, the screen will show a fringe pattern (Fig. 3).

The Wave Interference sim promotes analogy use with visual representations of water, sound, and light waves. Use of analogy involves mapping common ideas (such as frequency and amplitude) from across wave phenomena. In order for these analogs to be used productively for making sense of wave phenomena, users have to make comparisons between phenomena and draw conclusions based on these comparisons. The strategy employed in the sim is to use generic visual representations across different tabs as cues to promote analogy use. Figure 1 shows the generic representation for sound. Slight variations of this representation are used in the water and light tabs. In addition, alternate representations (such as the side view of water) are employed to cue real-world connections, promote the use of analogy, and serve to help students make meaning of the generic representations.

\section{THEORETICAL FRAMEWORK}

In this study, we focus on engaged exploration by students using sims, observed in the context of individual student interviews. First a student is presented with one or two open conceptual questions [13] before opening the sim [21]. These questions are constructed to be carefully balanced, sufficiently difficult, and open-ended so that students should be able to give a reasonable answer to the question after they have explored the sim. The goal of asking the open conceptual question is first to determine a student's initial under-

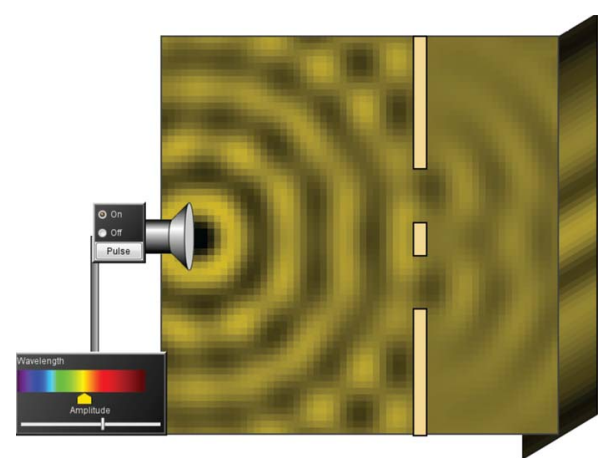

FIG. 3. (Color) Light wave with 2 slit barrier. Interference pattern is shown on the screen to the right. 
standing of topics covered in the sim. The second purpose of the open conceptual question is to direct students' initial interaction with the sim. Students often deviate from the original question, but if students continue to engage and explore while using the sim, it is considered a positive result. In this study, the metric for success is not the outcome of this process, i.e., whether students can answer certain questions or solve particular problems after the fact; rather, effectiveness of the sim is measured through observation of engaged exploration as it happens [22].

Note that by exploration, we do not mean what is often described as pure discovery, which may not provide sufficient scaffolding to support productive student activities $[23,24]$. The PhET sims include significant implicit scaffolding, which supports student engagement and inquiry. This scaffolding is implicit in that it consists of what the user can and cannot do as well as what the user is and is not shown. A significant part of implicit scaffolding can be described in terms of affordances and constraints, described below [25-27].

Affordances are what a user of a tool perceives as possible and productive action with that tool. Affordances can be properties of naturally occurring objects, for instance one could use a rock to pound nails into wood because the rock fits the hand and is quite rigid. However, humans recognize that a rock is fairly awkward for tasks like pounding nails. We therefore design hammers with unique features, i.e., affordances, such that they are particularly good tools for pounding nails. Similarly, we can build affordances into sims. For instance, users might be given control over the wavelength and amplitude of a light wave with sliders that look like realistic controls. In this way, users readily perceive that these controls are ways of adjusting parameters in the sim, are readily able to make these adjustments, and can observe the results. Another example is slowing of phenomena to within the limits of human perception, e.g., slowing the speed of a light wave so that the wave nature of light is perceivable by the user. In both cases, these features are designed so that students perceive the sims as engaging and are inclined to interact with the sim in productive ways.

Constraints restrict the actions that a user can take and, like affordances, can be purposefully built into tools. Constraints are extremely important for implicitly guiding the use of a tool since they restrict the user from taking inappropriate actions. Continuing the analogy of the hammer, one would never think to use a hammer to turn a screw. With sims, we often want to constrain actions that may be unproductive for engaged exploration. Examples of this would be restricting the range of the wavelength adjustment for light to the visible part of the spectrum. Allowing users to view ultraviolet or infrared wavelengths might be a distraction that hinders productive exploration, depending on the goals of a particular sim-based activity. Similarly, in Wave Interference users can move certain objects in one or two dimensions, but not three, and can activate one or two wave sources that are in-phase, but not more and not out-of-phase. These constraints keep sim users within a parameter space of the sim that is productive for exploration, and out of the range of parameters that are unnecessary and potentially confusing. Note that sims are not designed to address all possible learn- ing goals (e.g., water wave dispersion or out of phase incoherent light sources).

A cognitive affordance that is built into PhET sims is the use of analogy [28]. Analogy is generally described as the use of ideas from a familiar topic to make inferences about an unfamiliar topic $[14,20]$. Formal models treat an analogy as a mapping of ideas from one topic to another, akin to a mathematical mapping. For instance, ideas such as "oscillating," "adding," and "canceling" can map from water waves (a familiar topic) to light waves (which is less familiar) in order to explain interference of light. Notably, water and light waves are fairly broad topics with many complexities and nuances. Nonetheless, students with partial understandings of these topics can still use analogies to generate inferences and new ideas. Furthermore, students who are deeply engaged with a sim can build connections in real time and test these connections. Such is the case with students using Wave Interference, as we will demonstrate.

In the process of generating new ideas, student knowledge may be characterized as consisting of pieces $[29,30]$. These pieces of knowledge can be used to construct new understandings of unfamiliar phenomena if they are activated and applied appropriately. We can characterize a significant amount of this process as student use of analogy, which is supported in the sims through the use of multiple visual representations. In the Wave Interference sim, visual representations play two key roles in promoting analogy use by students: (1) cueing knowledge elements for sense making within a single topic, such as transverse wave motion of water waves, and (2) connecting knowledge elements across topics, such as constructive and destructive interference of water and light waves. The way visual representations are used in the sim is described in detail below.

In summary, affordances and constraints provide implicit guidance to students, driving productive activity. We suggest this may lessen the need for significant external guidance. This framework distinguishes engaged exploration with sims from other types of activities, such as heavily guided (i.e., cookbook) activities or pure discovery. Notably, sims are not meant to replace other pedagogical tools entirely. In the examples above, our intention is to highlight a primary strength of sims: educationally beneficial affordances and constraints can be built into sims that may not be easily accessible in the real world.

\section{METHODS}

This study uses qualitative data gathered from interviews in which students explore the Wave Interference sim for about $1 \mathrm{~h}$. We use exemplars from this data set to demonstrate cases of engaged exploration and use of analogy, as described above. In particular, we look for instances of affordances and constraints guiding productive exploration by students.

In these $1 \mathrm{~h}$ interviews, students are not expected to arrive at a complete and robust understanding of wave interference, rather, the goal is that they will begin to develop a framework about wave interference by noticing what is important and what is not important for understanding the phenom- 
enon. Wave interference is a fairly advanced and difficult topic, and the students we interviewed in this study had no formal instruction on the topic previously. Before using the Wave Interference sim, these students were questioned about interference phenomena but none of the students articulated the standard scientific explanation. However, these students constructed significant understanding of the topic through interaction with the sim and, while engaged with the sim, were able to articulate many of the ideas necessary for explaining interference phenomena.

Our research focuses on the following questions. Sims are designed with the intent of supporting students in making conceptual connections, and we are particularly interested in investigating whether students achieve these goals. (1) Can we identify cases of affordances and constraints driving productive, engaged exploration by students? (2) Do students notice and use connections between different wave phenomena to make sense of wave interference-that is, do students use the analogies built into the Wave Interference simulation? (3) Does student use of analogy appear to be supported by the particular representations used in the sim? (4) How do students make sense of these representations and does exploration help them make progress toward the standard scientific model of wave interference?

We seek to answer these questions with qualitative data from interviews with students using the Wave Interference simulation. Interviews were conducted with undergraduate students, recruited from a first-semester, algebra-based introductory physics course at a large university. This course is fairly traditional, with three lectures and a single laboratory section per week. All of the students had previously seen a few PhET sims as part of this course, but not the Wave Interference sim. Interference phenomena are not covered until the second semester of this course, so students had not yet been exposed to this material. The students were mostly science majors, but not in physics. Students were paid for their time (approximately $1 \mathrm{~h}$ ). These interviews were videotaped for later analysis. Video recording captured the student, interviewer, and the computer screen showing the sim.

The study began with six separate preliminary student interviews. Interviews began with the interviewer gathering background information such as major, year in school, and physics background of the student. In these preliminary interviews, students were asked an open conceptual question similar to the following: "If you have two light sources shining toward a screen, you can create what is called an interference pattern. How can you explain the pattern that you see on the screen?" Students were asked to give their best answers to this question before opening the sim. After answering, these students were presented with the Wave Interference sim and asked to use the sim to answer the question as best they could. The interviewer allowed the students to proceed at their own pace, occasionally interjecting prompts or questions to probe students' thinking. These interjections were usually minimal and clarifying in nature, such as, "what do you mean by that?," "can you explain that more?," or, "did you notice the tabs at the top?" On occasion, the interviewer might ask more specific conceptual questions. These prompts were unscripted and only provided after students had explored significantly on their own. Students were never explicitly told what to do with the sim.

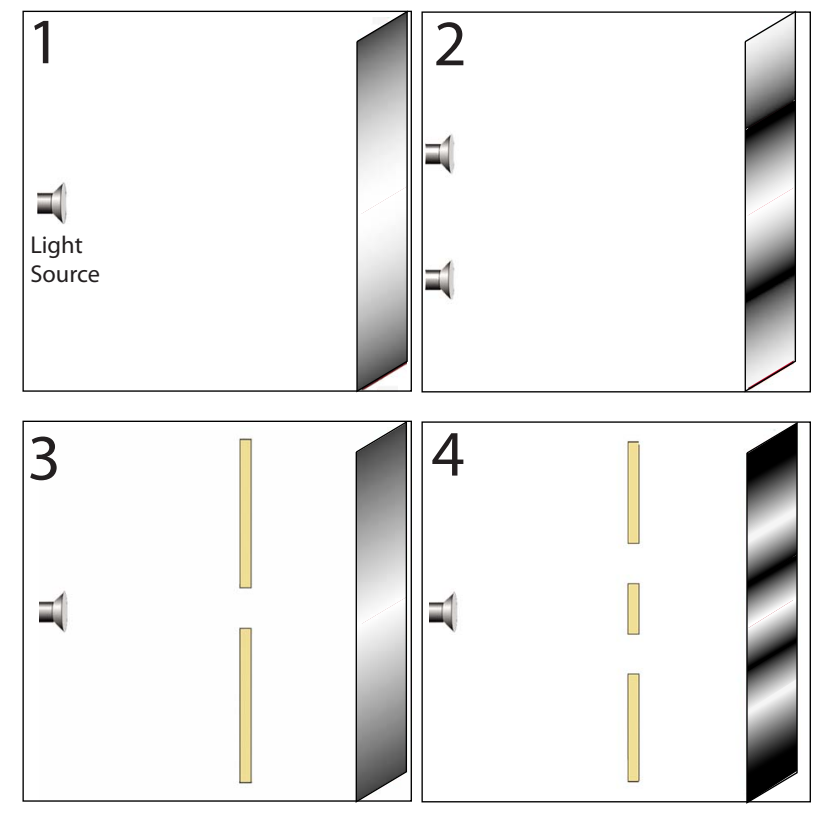

FIG. 4. (Color) Image of four "contrasting cases" used with open conceptual question for final two interviews.

With this protocol, students engaged with the sim to a significant degree, but there was some initial difficulty in that students were not familiar with interference patterns. With the final two students that were interviewed, the open conceptual question was modified to show students the interference pattern and present four contrasting cases [31] of light interference patterns (Fig. 4). Before starting the sim, students were presented with the picture in Fig. 4 on a sheet of paper and told that it showed light sources on the left, a screen on the right with an interference pattern, and barriers with one or two slits which let light through (cases 3 and 4). Students were asked to try and come up with a general model or explanation for how the patterns were created in the four cases. Students presented their initial ideas, and then started the sim and were told to use the sim as best they could to answer the challenge. While findings from all 8 interviews were similar in terms of student engagement and exploration, only the final two interviews adhered strictly to a protocol based on the open conceptual question based on Fig. 4. We therefore focus on results from these two final interviews below.

\section{RESULTS}

In this section we analyze transcript segments from interviews with students $\mathrm{S} 1$ and S2. S1 is a senior, majoring in math and philosophy, and had previously taken physics in high school. S2 is a sophomore majoring in biology and was retaking the algebra-based physics course after receiving a D in the course. S2 had no prior formal physics instruction. Neither student had previously received any formal instruction on wave interference. When presented with the open conceptual question about the four interference cases in Fig. 4 before opening the sim, both students' explanations clearly indicated that they did not have a good understanding of 
light interference. Common ideas were that the barriers "blocked" the light, or that the light "crossed" and gave the patterns; but, both S1 and S2 stated explicitly that they could not explain why cases 2 and 4 had three bright spots. Following this, students opened the Wave Interference sim. We present interview data from the point at which students began interacting with the sim below.

\section{Student 1 Interview}

After attempting to explain the four cases, S1 opened the sim and began to explore, starting with the water tab. After spending several minutes becoming familiar with the basic operation of the sim, such as moving sliders to make changes, S1 engaged in the first of many exploratory activities. S1 adjusted the frequency and amplitude of the water wave and activated the tape measure.

Without prompting by the interviewer, S1 said, "Out of curiosity, does changing the amplitude affect that [distance between circles; wavelength]." The interviewer did not respond. S1 then adjusted the amplitude of the water wave to be very small, then very large, and then paused the sim. Pausing freezes the wave representation shown in the wave pool. S1 used the tape measure to determine the distance between the circles, concluding, "No, it didn't appear to affect the length of it."

This short segment is just one example of the sort of exploration that students engage in when using the sim. This exploration follows a common pattern. First, some element of the sim draws the user's attention, leading the user to manipulate the element and see the result in real time. Upon seeing this result, the user generates further questions, and then explores the sim further in order to answer these questions. Note that this sort of productive exploration hinges on several affordances built into the sim: the adjustment of amplitude, the real-time change in representation in reaction to the adjustment, the availability of a tool (the tape measure) that allows for the measurement of length, and the ability to pause the sim. Built-in constraints also support engaged exploration: with a real faucet, increasing the water flow in order to generate a larger amplitude would necessarily increase the frequency of drips. However, this is an artifact of faucets, not a general property of waves. In the sim, the faucet is designed such that increasing the water flow affects the drip size (and thus the amplitude of the waves), but is kept from affecting the frequency. This constraint on the effect of amplitude supports students in generating the idea that amplitude and frequency are independent quantities of a wave.

S1 spent several minutes further exploring the sim, manipulating controls, changing to the side view of water, and using the detector to probe different areas of the wave pool. About fourteen minutes into the interview, still working in the water wave tab, S1 activated the option for two faucets and saw that a different pattern was created in the wave pool. This pattern (Fig. 5) shows light and dark circles moving away from the sources, as well as fuzzy lines that represent nodal lines where the wave amplitude is zero. S1 used the detector to see that the water height was not changing along

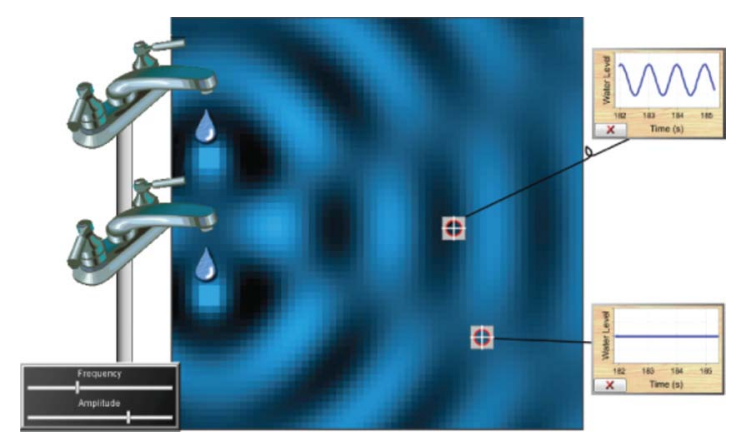

FIG. 5. (Color) Two source interference of water waves. Fuzzy lines represent nodal lines where wave amplitude is zero. Detectors are placed in an area of large wave activity and on a nodal line, resulting in a large amplitude curve and a flat line on the detector graphs, respectively.

the nodal lines, but oscillated in other areas. Noticing these features, S1 said,

S1: I wonder what defines these lines [pointing along the nodal lines]...no wave activity...right in between them is where the waves are the biggest, and here they mellow out. So what I was wondering is how you determine that.

S1 then spent several minutes moving the detector around the wave pool, then changed back to a single faucet and activated a barrier with a single slit. This creates a diffraction pattern (similar to interference, but for a single slit) emanating from the slit, which S1 continued to examine with the detector. The following discussion then ensued between S1 and the interviewer (I):

S1: That's intriguing. You have the same thing again...right here in the middle, just like when we had two drips, they interfered with each other and made... and calmed the water.

I: So what do you mean when you say interfere?

S1: I'm not sure. The waves are going in the same spot and the end result is a shallower wave...with a lower amplitude. Here, the amplitude here on these two lines is, um, a height without numbers, and over here it's less. And so if you put a screen out here, out here, you'd have, um, two white spots, and then a darker spot in between them.

Here, S1 makes a comparison between the pattern created by two drips and the pattern created by the slit. Initially, the language that $\mathrm{S} 1$ uses is very specific to water- $\mathrm{S} 1$ talks about "where the waves are the biggest, and here they mellow out." While S1 uses the word "amplitude," which is not specific to water, here S1 explicitly relates amplitude to "shallower." S1 is exploring the sim and trying to make sense of the representations of water waves. In using the detector, S1 is coordinating two representations of the water wave-the wave pool ripples and the sine wave in the detector-and is using the detector representation to make sense of the wave pool representation.

$\mathrm{S} 1$ generates two spontaneous analogies in the segments above. First, with the single slit, S1 says "you have the same 
thing again," relating the interference pattern to the twosource case. Note that S1 sets up these two situations and then notices the similarity between them. S1 also makes a spontaneous analogical connection between water and light. In the last line, S1 says "if you put a screen out here," (Note that there is no screen shown in the water tab.) S1 is using the representation shown in the water tab to make sense of the initial question about light, in which only the final interference pattern on the screen is shown.

Next, S1 activated two slits and continued in a similar process of probing with the detector, manipulating the sim, and observing the effects. During this time, S1 noted how the two slits created an interference pattern similar to two sources or a single slit.

$\mathrm{S} 1$ continued to explore the water tab, and at about thirtyfive minutes, the interviewer said, "Did you notice the tabs up at the top?" S1 said, "No, I was just playing with water," and immediately switched to the sound tab. S1 spent a few minutes exploring sound, and then at about forty minutes into the interview, $\mathrm{S} 1$ began exploring the light tab.

S1 first activated two light sources, then paused the sim to examine the pattern shown.

S1: Again, we have the lines where the waves don't happen, and that's why we've got darkness in the middle. Because just like with the water, the waves are interfering with each other somehow. And so we'll have another, um, white spot, or black spot out here and one out here as well.

Here, S1 is describing what is seen in the wave pool and spontaneously makes a reference to water, based on the light wave interference pattern shown. S1 is making another analogical comparison between water and light, this time comparing two tabs of the sim (whereas earlier S1 compared water waves in the sim to the light interference pattern on a sheet of paper). Water serves as a familiar resource for S1 to generate ideas about light. Though S1 had made a comparison between water and light earlier, the representation using bright and dark areas in the wave pool appears to be critical for S1 noticing the specific similarities between water and light waves (e.g., referring to the "white spot, or black spot"). S1 continued examining the interference pattern, and the interviewer prompts $\mathrm{S} 1$.

I: So what do you think is going on out there?

S1: What do you mean?

I: Um, why do you think you get these different patterns? I: How would you explain these different cases now?

S1: Um, I think that the, like I've been saying it the whole time, the waves are interfering with each other. And maybe it's that one wave is here [raises right hand up, palm down] and the other one is here [puts left hand lower, palm down] and they crash into each other [bringing hands together], they... [hitting hands together several times]. I don't know, they...[shrugs]"' I: What would give you that idea?

S1: Um, I'm not really sure. I guess that when they, when the waves interact, um, the like parts of the wave...it doesn't matter why you've got separate waves. Whether you've got two lights and the waves are interacting, or two drips, or two speakers or whatever and the waves are interacting. Or if you've got a barrier and...um... and now we've got waves coming off, separate waves coming off of each of these slits, and we added each of these slits...so the different waves interact with each other [bringing hands together] and you see that they interact, and they've been doing that the whole time, but um...I just would want to know, like, exactly what that interaction is [bringing hands together again]. What happens there."

S1 first explains that "the waves are interfering with each other" and makes a gesture (another representation) of something being up and something being down coming together. $\mathrm{S} 1$ is not able to articulate these ideas very well in words, but S1's gestures are suggestive of waves cancelling akin to destructive interference. Note that $\mathrm{S} 1$ ends the last statement by suggesting further questions to be answered. While this indicates that S1's ideas about interference are tenuous, this sort of questioning by students is exemplary of engaged exploration since it indicates that students are choosing and exploring their own inquiry path. Furthermore, S1 generates many useful ideas about wave interference and may be in the process of developing a coherent conceptual framework of the phenomena. This process of developing a framework is important for students to make sense of content they encounter in the future [32,33]. Along these lines, we will soon see that $\mathrm{S} 1$ is able to demonstrate a fairly robust understanding of wave interference.

$\mathrm{S} 1$ is also beginning to generalize the idea of waves. S1 says, "it doesn't matter why you've got separate waves," and the rest of S1's talk makes it clear what is meant is that it does not matter what specific wave phenomenon is being described. S1 is generalizing, saying that water, sound, and light waves all behave the same way.

I: So what would be your best explanation for what's going on at the dark spots and what's going on at the light spots on the screen?"

S1: The, um, amplitude of the wave determines the brightness of the, um, the spot on the screen. And so here, where the interfering waves, like before the barrier has kind of broken up the wave a little bit, so you just get what's coming to a slit, and so they interfere with each other and so the amplitude is smaller. You get, um, a darker spot. But, like you've got light here and here and here [pointing at the screen], um, just like when we were talking with the water [switches to the water tab]. Where we had the two slits and they interfered with each other and the line between them. But then they had the space in the middle.

$\mathrm{S} 1$ continues to draw on water to explain what is happening with light. However, now S1 actually switches to the water tab, using this element of the sim as a resource for explaining his reasoning. This is a significant shift in how S1 is using the sim. The activity is no longer a matter of interaction between student and $\operatorname{sim}-\mathrm{S} 1$ is now using the sim as a tool for communicating with the interviewer. In a sense, the sim provides not only scaffolding for the student's explora- 
tion, but also for the social interactions between student and interviewer. Continuing the discussion,

I: So what do you think is going on in that space in the middle?

S1: Here? [points to bright spot centered between the slits on the screen] Um, the wave...if we go with the hypothesis where when they collide with each other they kind of equal out [brings hands up and down then together] then that space in the middle would be where they're both cresting [brings both hands up together] or where they're both [brings hands down together]. Like, the wavelength is the same, so where they're both coalescing [makes wave motion with both hands together]. And this space between those, where you've got the dark spot, is where they, um, conflict with each other and they cancel each other out [slaps hands together]. Would be my guess. [laughs]

Here, S1's talk suggests "colliding" or "coalescing" as the mechanism of interference, but his gesture suggests adding or canceling along the lines of constructive and destructive interference. At the end of this segment S1 does use the word "cancel," but then comments that this is a guess. What we see is S1 using an array of reasoning strategies, drawing on the sim as a reasoning resource. This final explanation, while shaky for $\mathrm{S} 1$, is a result of a series of dynamic interactions with the sim, analogical comparisons, and meaning derived from the representations in the sim. Importantly, many of the analogical comparisons hinged on S1 seeing similar representations in different situations. This is exactly the sort of student engagement that the Wave Interference sim was designed to promote.

\section{Student 2 Interview}

S2 started exploring with the sim by immediately selecting the light tab. (Later S2 returned to the water and sound tabs, as we will discuss below.) During the first few minutes, $\mathrm{S} 2$ explored by activating the screen, changing the wavelength of light and observing the response of the sim. S2 then activated a barrier with a single slit, observed the resulting representation, then changed to two slits. At this point S2 said,

S2: It breaks it, so you get the three ripples hit. Makes the three spots of light. When it hits the middle piece, instead of coming at it like one piece, it splits it [the wave] in half and then they come back together.

S2 then removed the barrier and activated two light sources, which produces a similar interference pattern. Upon observing this, S2 tried again to describe how the pattern formed.

S2: When they combine they hit each other, they form and make that ripple effect. They come out in circles, and when they collide, and when they collide they combine into one wavelength instead of separate ones... when they collide you're going to lose energy, but I don't know why the wavelength would change shapes.
Several things are happening in this segment. First, S2 is trying to explain the patterns both on the screen and also in the wave pool. S2 is able to do so because the sim includes a representation of the light wave in the space between the source and screen. This wave pattern is a visual representation that is simply not possible in the real world, and is a critical affordance of the sim. Second, S2 is able to set up different cases (two slits vs two sources) and observe the similar effects on the interference pattern. Specifically, S2 is able to create two-source interference with light, which is extremely difficult to achieve with real equipment. The sim allows this situation to be set up quite easily. Nonetheless, S2 does not appear to know why these patterns result. S2's best guesses include ideas like "hitting" and "colliding," but there is no hint of ideas like "adding" or "canceling," which are key ideas for explaining the interference patterns.

S2 spent a significant amount of time exploring the light tab, mostly changing parameters and observing the resulting patterns. About 25 min into the interview, still on the light tab, S2 activated the detector and began moving it around the wave pool. The detector in the light tab shows a graph of "Electric field" and S2 was able to use the detector to make sense of the "ripples" in the wave pool.

S2: So, on this [detector], it has the electric field. And where the two wavelengths combine and hit, the period of the graph [in the detector], from top to bottom is higher than when they recombine. Where it's almost a straight line [putting detector in a nodal line].

In the wave pool, there are very bright and dark areas, representing large oscillations of the light wave, and there are fuzzy lines in between, representing areas where the oscillations are very small or zero. Previously, S2 did not know what the fuzzy areas were representing, but through use of the detector, S2 was able to explore and coordinate the ripples representation with the graph in the detector. Once this was done, S2 put new meaning to the representation in the wave pool-specifically, the meaning of the fuzzy areas "where it's almost a straight line." Again, the detector is purely a construct of the sim that does not have a simple real-world counterpart. The detector allows S2 to make meaning of the representation in the pool, but it is also constrained in that it only measures the vertical oscillations of the electric field in the plane of the wave pool. Even if such an instrument existed in a real laboratory setting, it would be difficult for a student to measure the field oscillations so precisely and would present significant barriers to realizing the key ideas about light waves necessary for understanding interference. In the sim, visual representations make the electric field visible and time is slowed so that oscillations in the field can be seen and measured. With these representational affordances built into the sim, the detector becomes a highly productive tool, allowing users to readily measure the field strength at various locations.

An additional representational affordance is the way the wave pattern cues students as to where to investigate and measure in order to see important features of the pattern. The patterns are just complex enough so that students perceive that there is useful information to be explored, but simplified enough that the information is readily measurable. The sim- 
plified nature of the representation also constrains users by reducing "noise," that is, extraneous visual information that is not relevant to the sim learning goals. The fact that the detector gives users a way of making sense of the light wave, but limits users to just the information that is necessary, is a perfect example of a good balance between affordances and constraints built into the sim. Put another way, the challenge in this activity is to figure out why waves interfere, and the sim provides just the right amount of scaffolding such that this challenge is balanced between too easy (and therefore not interesting) and too difficult (and therefore frustrating). The result of these design choices is that students focus on the essential features for building understanding of wave interference: light and dark areas and areas that make "fuzzy lines" through the pattern.

Like S1, S2 made analogical connections between different wave phenomena, which supported S2's exploration. About 30 min into the interview, S2 switched from the light tab to the sound tab. S2 created an interference pattern with two speakers, then switched to the particles view and began to comment on what was shown.

S2: [I'm] still trying to figure out why it breaks. Cause they're doing the same thing that the light did, and they're breaking, when the wavelength breaks [splits at the barrier], it causes it to change shapes.

I: What's the same about them?

S2: The way they, um, when they combine they don't stay the same straight pattern that it would. And so it's hitting differently on the wall. It's hitting on the three spots instead of just one.

We note that S2 initiated the idea that sound and light were similar in the patterns shown in the wave pool. S2 still cannot quite explain these patterns, but the analogical connections are beginning to take shape. S2 next switched to the water tab and created an interference pattern with two faucets, then activated the detector and began examining the water waves with the detector probe.

S2: So here I have the water dripping in, and I have it set so it'll drip really fast, the frequency is higher. And, like on the light waves, they break up and they hit the wall differently. And here the water level is the smallest [moving the probe to a nodal line], where the light wave had the smallest electric field. And the same thing, the water level is highest when it first comes out of the spout [moving the probe near to one of the faucets].

I: So what do you think is causing that pattern here?

S2: When it first comes out, it has a higher velocity so the wave is going to have a higher impact on it, when it hits the water. Which causes the water to have a higher water level. And as it goes closer to the wall, it loses energy and so the waves aren't as high. So the water level decreases. So I guess if you applied that to the light [switches to light tab], it would kind of be the same thing I guess. When the light first comes out of the light bulb, it would be at its highest intensity and moving the fastest, and so when it gets closer to the wall it's slowing down. And then it won't, as it slows down it loses energy?
S2 continued to explore the water, sound, and light tabs with the detector. S2 made several more references to the three phenomena being similar, activated many pieces of knowledge about interference and made connections between phenomena. About $45 \mathrm{~min}$ in, the interviewer decided to probe more specifically what ideas S2 had generated by using the sim.

I: In the water one, what do you think the light places and the dark places are?

S2: It's the water level.

I: Which is which?

S2: [first taking some time to explore with the detector]

The dark places are going to be the deeper. I think the

light ones are going to be like the top of the wave, and

the dark ones are the in between them.

Notice that in the last line, S2 did not answer right away. S2 took time to use the detector, moving the probe around the wave pool to find an answer, then responded. S2 had been doing a similar exploration with the detector with water, sound, and light throughout the interview, and so at this point was well prepared and knew what action was needed to do in order to answer the interviewer's question.

About 50 min in, S2 was exploring the water tab when the interviewer began to ask a series of probing questions.

I: What do you think would happen if two waves were coming along and one of them was going up and one was going down...

S2: And they hit?

I: ... and they hit.

S2: It would go back to zero. They would neutralize each other, I guess.

I: And so, does that help you think about this at all?

S2: [switches to light tab] So, when they collide, the amplitudes are opposite so one is at the top, one is at the bottom [raises one hand up, one hand down]. And when they collide they zero out [brings hands together] and that's why you get the, you don't have the light.

A few moments later, the interviewer asked $\mathrm{S} 2$ to explain again how the pattern was formed, and what S2 did is telling. S2 was in the light tab, but switched to water to begin an explanation, grounding the ideas in water waves.

I: So can you explain it to me again, the way it makes sense to you.

S2: So, with the water [switches to water tab], the two waves they come at each other and one's high and one's down low. When they collide they cancel out and they go back down to the normal water level. And so for the light, when they do that [switches to light], it would be the same thing with the wavelengths. The one wavelength would be up high, the one would be on the bottom, and they collide. And so when they collide they cancel out. When they cancel out you don't get the light any more. You can't see the wavelength if it's zero.

At this point, S2 is able to give a very good explanation for the interference pattern. Note particularly how S2 uses water to explain the ideas, even to the interviewer who pre- 
sumably understands the phenomena. This is similar to S1's use of water in an explaining capacity. S2 also fluidly switches between water and sound tabs in the sim, using the sim as a resource for explanation. The sim provides a common representation of phenomena shared between $\mathrm{S} 2$ and the interviewer, and S2 uses these quite readily to communicate the ideas.

It is notable that both S1 and S2 demonstrated an expertlike ability to move between representations both when generating and explaining their ideas. We might not expect students to use representations this way [34], [35], especially with a topic that is unfamiliar and complex like wave interference. In our observations, students do act in such expertlike ways, and we credit this to the design of the sim as well as the context of the activity; rather than being guided through a set of questions, these students were asked to make sense of wave interference phenomena in order to explain a set of results (as depicted in the open conceptual question). We therefore see evidence that students can be quite capable of coordinating and making meaning from multiple representations when provided with tools and contexts which support sense making and engaged exploration.

\section{DISCUSSION}

Educators may have a number of goals in mind when using computer simulations in educational activities. In this study, we focus on the goal of student engagement, particularly exploring and investigating similar to a scientist. The PhET sims are designed to promote and facilitate this sort of engagement, and we have observed students using sims in this way in hundreds of interviews. We demonstrate this process in detail. S1 and S2 both engaged with the Wave Interference sim, beginning with the open conceptual question, but then continued to ask their own questions and investigate interference phenomena along their own paths. Although they took very different paths initially, S1 starting with water while S2 started with light, both students used the sim in similar ways overall and made significant progress in developing a conceptual framework for interference phenomena. Notably, for S2, interaction with the interviewer was helpful for maintaining engaged exploration, whereas S1 appeared to need less direct interaction with the interviewer. However, this interaction with S2 consisted of prompts to elaborate or think more deeply. Following these prompts, the interviewer gave S2 control over what to do next. In other words, S2 was still in control of how sense making and exploration would proceed. In numerous interviews with students using sims, we have observed similar variation in the degree of interviewer interaction that students need, but students are almost always given ultimate control over how their interaction and sense making proceeds.

We need to emphasize that these students' interaction with the sim was not guided by a preset series of questions, as might be commonly used in a science laboratory activity [36]. These students largely led their own investigation, a critical element of what it means to "act like a scientist" and of what we term "engaged exploration." Adams, Paulson, and Wieman [13] suggest that students who are given a sim and a set of guided questions tend to only answer those questions and do not explore elements of the sim that are not specifically covered by the questions. On the other hand, they found that students who were given two open conceptual questions before using that same sim investigated all of the same elements of the sim, but also additional elements that the guided students did not. Hence, guiding students too much may "short circuit" exploration and limit how students engage with a sim.

Practicing scientists rarely follow a set of directions when investigating phenomena. However, there are important differences between how practicing scientists investigate research questions and how students engage with educational materials. Students have less experience in this sort of investigation compared to practicing scientists, and thus need more scaffolding in order to engage, ask productive questions, and explore unfamiliar phenomena, such as wave interference. We suggest that providing this scaffolding in the form of affordances and constraints, implicitly built into the tools rather than explicitly provided as a series of instructions, supports more authentic and productive scientific inquiry by students.

This study demonstrates what engaged exploration looks like, and how specific features of sims can support this type of student engagement. Affordances give students a substantial amount of control and flexibility to direct their exploration, and can show representations of phenomena that are not available in the real world. Sims also provide affordances in the form of analogies that serve as grounding for students to make sense of new ideas. Experts often generate analogies spontaneously to make sense of unfamiliar phenomena [37], but students may not [38]. One reason for this is that students do not understand the unfamiliar phenomena, such as light, well enough to know which analogies would be useful. We overcome this hurdle by providing useful analogies directly to students in the sim. Students investigate the useful parts of each analogy by engaging with the sim, for instance, by exploring the water tab. We employ similar representations across different phenomena which cue students to make connections and build understanding through analogical reasoning.

Especially important to making affordances useful is that students readily perceive that they can take certain actions and make sense of the phenomena with the sim. This often means using realistic objects, such as faucets or speakers, as grounding or hooks for engaged exploration and sense making. For instance, in one of the six preliminary interviews, a student had been trying to make sense of light and having significant difficulty. When she later explored the water tab, she said it made much more sense "because, you know, it's water." Our interpretation of this statement is that water is not only more conceptually concrete for this student, but she also believes she can make sense of water waves, whereas she was less sure of herself when trying to make sense of light. In this way, concrete and familiar contexts may promote a sense of self efficacy [39] for students, whereas overly abstract contexts may have the opposite effect if not accompanied by corresponding concrete contexts [40]. We have observed this effect in many interviews with students using sims. 
Constraints play a significant role in scaffolding student exploration by reducing cognitive demands and keeping students from creating situations which are unnecessarily complicated or distracting from the main educational goals of the sim. The frequencies of water, sound, and light waves are limited to those that are pedagogically useful for exploring interference phenomena in the context of the sim. Users can activate two sources or slits, but not more, which would create complicated interference patterns that would be difficult for students to interpret. The detector is constrained to measure the waves in a single plane (e.g., as opposed to above or below the water surface), and constraints of this type are extremely valuable as students are building a conceptual framework for understanding and communicating about phenomena.

We have shown that the Wave Interference sim provided the scaffolding necessary for these interviewed students to generate a useful conceptual framework. Once students have begun to develop this framework, instructors can build on students' ideas to further explore details and complexities of phenomena. Like any pedagogical tool, sims are meant to be a useful supplement to, not a replacement for, high-quality teaching.

In general, sim design involves achieving a careful balance between what users can do with a sim and what they cannot. Affordances and constraints complement each other and provide a useful framework for identifying the critical features that make a sim productive for student engagement. Importantly, this sort of implicit scaffolding, combined with user control, flexibility of use, and opportunities for productive play, sets up balanced challenges which motivate students to explore the sim and make sense of the phenomena. Affordances and constraints provide a useful framework for sim design in general. However, each individual sim is unique and requires an iterative process of design and testing with students.

\section{CONCLUSION}

In a series of interviews, we investigated student engaged exploration using computer simulations. We have observed this type of engagement in numerous interviews with students using PhET sims, and here we examine this process in more detail. Fostering engaged exploration depends on building implicit scaffolding into education materials. In the sims, this scaffolding consists of affordances, productive constraints, analogies, and representations of phenomena that allow students to construct understanding of science topics.

In the Wave Interference sim, students can explore the sim freely, choosing what order to use the representations. Since no two students use a sim in exactly the same way, this flexibility can actually be advantageous, and in practice we find that students tend to explore the sim in ways that are intuitive for them. This freedom to proceed along their own path is critical to students exploring like a scientist. However, this exploration is implicitly guided by building sims so that students are likely to interact with certain sim elements first, and more likely to see some visual representations before others. In this way, students tend to make connections between representations and arrive at interpretations that are useful for understanding scientific ideas. Furthermore, representations are presented in such a way that student interpretations of these representations, and hence their intuitions about phenomena, are productive for generating ideas about the science.

By interacting with the sim, students coordinate across multiple representations of water, sound, and light waves, and use analogies to develop a general framework for understanding wave interference. The effectiveness of this approach is demonstrated with the Wave Interference sim. Though students in this study take different paths, sometimes starting with water, sometimes with light, and taking different actions in between, S1 and S2 follow certain patterns of exploration which are similar and come to essentially similar conceptual understandings of the phenomena. These students proceeded to explore the sim, ask and answer their own questions beyond the initial open conceptual question, and investigate wave interference much the way a scientist would investigate some phenomenon. Balanced challenges that are created by implicit scaffolding in the form of affordances, productive constraints, analogies, and use of multiple representations are critical to supporting this type of student engagement. It is a testament to the design of the sims and the power of this technology, when well designed, that this type of self-directed student inquiry can be realized.

\section{ACKNOWLEDGMENTS}

This work is supported by NSF Grant No. DUE-0817582, the William \& Flora Hewlett Foundation, C. Wieman and S. Gilbert, JILA Center for AMO Physics, King Saud University and the University of Colorado. Thanks to the PhET team and the PER group at Colorado, especially to Sam Reid the software developer that coded Wave Interference, and to Carl E. Wieman.
[1] A. A. diSessa, Changing Minds (MIT Press, Cambridge, MA, 2000).

[2] M. C. Linn and S. Hsi, Computers, Teaching, Peers: Science Learning Partners (Lawrence Erlbaum Associates, Mahwah, NJ 2000).

[3] S. Papert, Mindstorms: Children, Computers, and Powerful Ideas (Basic Books, Inc, 1993).
[4] http://phet.colorado.edu

[5] W. K. Adams, S. Reid, R. LeMaster, S. B. McKagan, K. K. Perkins, M. Dubson, and C. E. Wieman, A Study of Educational Simulations Part I-Engagement and Learning, J. Interact. Learn. Res. 19, 397 (2008).

[6] W. K. Adams, S. Reid, S. B. McKagan, K. K. Perkins, M. Dubson, and C. E. Wieman, A Study of Educational Simula- 
tions Part II-Interface Design, J. Interact. Learn. Res. 19, 551 (2008).

[7] N. D. Finkelstein, W. K. Adams, C. J. Keller, K. K. Perkins, C. E. Wieman, and PhET Team, "High tech tools for teaching physics: the Physics Education Technology Project, Journal of Online Teaching and Learning 2, No. 3, 109 (2006).

[8] K. K. Perkins, W. K. Adams, M. Dubson, N. D. Finkelstein, S. Reid, C. E. Wieman, and R. LeMaster, PhET: Interactive simulations for teaching and learning physics, Phys. Teach. 44, 18 2006.

[9] D. Bodemer, R. Ploetzner, I. Feuerlein, and H. Spada, The active integration of information during learning with dynamic and interactive visualizations, Learn. Instr. 14, 325 (2004).

[10] J. van der Meij and T. de Jong, Supporting students' learning with multiple representations in a dynamic simulation-based environment, Learn. Instr. 16, 199 (2006).

[11] M. R. Lepper and T. W. Malone, Intrinsic motivation and instructional effectivness in computer-based education, Aptitude, Learning, and Instruction, edited by R. E. Snow and M. J. Farr (Lawrence Erlbaum Associates, Hillsdale, NJ, 1987.

[12] T. W. Malone, What makes computer games fun?, BYTE 6, 258 (1981).

[13] W. K. Adams, A. Paulson, and C. E. Wieman, What levels of guidance elicit engaged exploration with interacive simulations, Proceedings of the 2007 Physics Educatoin Research Conference (AIP Press, New York, 2008.

[14] D. Gentner and D. R. Gentner, in Mental Models, edited by D. Gentner and D. R. Gentner (Lawrence Erlbaum Associates, Hillsdale, NJ, 1983).

[15] N. S. Podolefsky and N. D. Finkelstein, Analogical scaffolding and the learning of abstract ideas in physics: An example from electromagnetic waves, Phys. Rev. ST Phys. Educ. Res. 3, 010109 (2007).

[16] N. S. Podolefsky and N. D. Finkelstein, Analogical scaffolding and the learning of abstract ideas in physics: Empirical studies, Phys. Rev. ST Phys. Educ. Res. 3, 020104 (2007).

[17] E. Wong, Understanding the generative capacity of analogies as a tool for explanation, J. Res. Sci. Teach. 30, 1259 (2006).

[18] D. Brown and J. Clement, Overcoming misconceptions via analogical reasoning: abstract transfer versus explanatory model construction, Instr. Sci. 18, 237 (1989).

[19] D. Gentner, Structure-mapping: A theoretical framework for analogy, Cogn. Sci. 7, 155 (1983).

[20] K. J. Holyoak and P. Thagard, The analogical mind, Am. Psychol. 52, 35 (1997).

[21] J. S. Krajcik, P. C. Blumenfeld, R. W. Marx, and E. Soloway, A collaborative model for helping middle grade science teachers learn project-based instruction, Elem. Sch. J. 94, No. 5483 (1994).

[22] A. Sfard, When the rules of discourse change, but nobody tells you: Makking sense of mathematics learning from a commognitive standpoint, J. Learn. Sci. 14, 565 (2007).
[23] C. Hmelo-Silver, R. G. Duncan, and C. A. Chinn, Scaffolding and achievement in problem-based and inquiry learning: A response to Kirshner, Sweller, and Clark (2006), Educ. Psychol. 42, 99 (2007).

[24] P. A. Kirschner, J. Sweller, and R. E. Clark, Why minimal guidance during instruction does not work: An analysis of the failure of constructivist, discovery, problem-based, experiential, and inquiry-based teaching, Educ. Psychol. 41, 75 (2006).

[25] J. J. Gibson, in Perceiving, Acting, and Knowing: Toward an Ecological Psychology, edited by R. Shaw and J. Bransford (Lawrence Erlbaum Associates, Hillsdale, NJ, 1977).

[26] D. Norman, The Psychology of Everyday Things (Basic Books, Inc, New York, NY, 1988).

[27] W. M. Roth, C. Woszczyna, and G. Smith, Affordances and constraints of computers in science education, J. Res. Sci. Teach. 33, 995 (1996).

[28] "In our view, all sims have analogies built in, whether explicit or implicit. How.."

[29] A. A. diSessa, in Constructivism in the Computer Age, edited by G. Forman and P. B. Pufall (Lawrence Erlbaum Associates, Hillsdale, NJ, 1988).

[30] D. Hammer, A. Elby, R. E. Scherr, and E. F. Redish, in Transfer of Learning from a Modern Multidiscilinary Perspective, edited by J. Mestre (Information Age Publishing, Greenwich, CT, 2005), pp. 89-120.

[31] D. L. Schwartz, R. Lindgren, and S. Lewis, in Constructivist Instruction: Success or Failure?, edited by T. Duffy and S. Tobias.

[32] J. D. Bransford and D. L. Schwartz, Rethinking transfer: A simple proposal with multiple implications, Rev. Educ. Res. 24, 61 (1999).

[33] A. A. diSessa and B. L. Sherin, What changes in conceptual change? Int. J. Sci. Educ. 20, 1155 (1998).

[34] S. R. Goldman, Learning in complex domains: when and why do multiple representations help? Learn. Instr. 13, 239 (2003).

[35] R. Kozma, The material features of multiple representations and their cognitive and social affordances for science understanding, Learn. Instr. 13, 205 (2003).

[36] S. R. Singer, M. L. Hilton, H. A. Schweingruber, National Research Council, America's Lab Report (National Academies Press, Washington, D.C, 2006).

[37] J. Clement, Observed methods for generating analogies in scientific problem solving, Cogn. Sci. 12, 563 (1988).

[38] C. Sandifer, Spontaneous student generated analogies, Proceedings of the 2003 Physics Education Research Conference (AIP Press, New York, 2003).

[39] A. Bandura, Self-efficacy: Toward a unifying theory of behavioral change, Psychol. Rev. 84, 191 (1977).

[40] D. I. Cordova and M. R. Lepper, Intrinsic motivation and the process of learning: Beneficial effects of contextualization, personalization, and choice, J. Educ. Psychol. 88, 715 (1996). 